\title{
Quantum dots in carbon nanotubes
}

\author{
Sami Sapmaz, Pablo Jarillo-Herrero, Leo P Kouwenhoven and \\ Herre $S$ J van der Zant
}

Kavli Institute of Nanoscience, Delft University of Technology, PO Box 5046, 2600 GA Delft, The Netherlands

E-mail: sami@qt.tn.tudelft.nl

Received 14 March 2006

Published 17 October 2006

Online at stacks.iop.org/SST/21/S52

\begin{abstract}
In this overview paper, we present low-temperature electronic transport measurements of carbon nanotube quantum dots with a back gate. In a semiconducting tube, charge carriers could be completely depleted. The addition energy and the excitation spectrum have been studied as a function of the number of charges (electrons or holes), one by one. We observe electron-hole symmetry, which is a direct consequence of the symmetric band structure of the nanotube. The excitation spectrum for metallic nanotubes exhibits four-fold shell filling and is completely described by an extended constant-interaction model. Furthermore, nanotubes with a four-fold shell structure are investigated in a parallel magnetic field. The magnetic field induces a large splitting between the two orbital states of each shell, demonstrating their opposite magnetic moment and determining transitions in the spin and orbital configuration of the quantum dot ground state. Also, a small coupling is found between orbitals with opposite magnetic moments leading to anti-crossing behaviour at zero field. Current-voltage characteristics of suspended carbon nanotube quantum dots show an additional series of steps equally spaced in voltage. The energy scale of this harmonic, low-energy excitation spectrum is consistent with that of the longitudinal low- $k$ phonon mode (stretching mode) in the nanotube. Finally, we report on a fully tunable carbon nanotube double quantum dot. We perform inelastic transport spectroscopy via the excited states in the double quantum dot, a necessary step towards the implementation of new microwave-based experiments for quantum information technology.
\end{abstract}

(Some figures in this article are in colour only in the electronic version)

\section{Introduction}

Since their discovery [1], carbon nanotubes (CNTs) have emerged as prototypical one-dimensional conductors [2]. A continuous improvement in device fabrication and CNT quality has enabled the recent observation of many different quantum phenomena, e.g. Luttinger liquid behaviour [5], Fabry-Perot interferences [6], Kondo effect [7, 8], influence of vibrations $[9,10]$ and effects of superconductivity [11-13]. At low temperatures, CNT devices form quantum dots (QDs) where single-electron charging and energy level quantization effects dominate [3, 4]. In this paper, we focus on lowtemperature electron transport in CNT QDs. We start with the most basic three-terminal field effect transistor type geometry on insulating substrates. We continue our investigation with suspended CNTs (to study effects of vibrations) and finish with more sophisticated CNT structures to form double QDs.

\section{Fabrication}

Nanotubes are grown/deposited on the top of oxidized silicon substrates. The Si substrates are highly doped (p-doped in our case) so that they remain conductive at low temperatures and can serve as a back gate in our devices. The thickness of the thermally grown oxide is typically $\sim 250 \mathrm{~nm}$ and isolates the devices from the back gate. A set of markers is necessary to later locate the position of the nanotubes and for the fabrication of the electrodes. These include a 


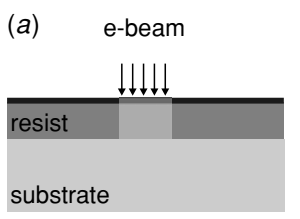

(c) Metal evaporation

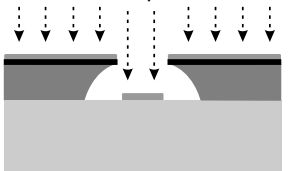

(b)
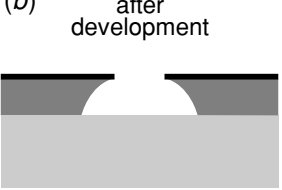

(d) After lift-off

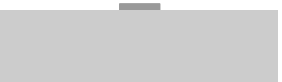

Figure 1. Schematic electron beam lithography cycle. (a) Double layer of organic resist is spun on a substrate and a predesigned pattern is irradiated with a beam of electrons. (b) After development, an opening is left in the resist. (c) Metal is evaporated on the top of the substrate + remaining resist. $(d)$ The remaining resist is removed and the metal is left at the predesigned positions.

set of electron beam lithography alignment markers (e-beam markers) and atomic force microscopy (AFM) markers. The patterning of these markers requires one e-beam lithography 'cycle' (figure 1), which consists of spinning a double layer of e-beam resist, e-beam lithography, development, metal evaporation and lift-off. The bottom layer of resist (poly-methyl methacrylate (PMMA) 350K 3\% in chlorobenzene) is thicker and more sensitive to e-beam radiation; it serves as a spacer and ensures a proper lift-off. The top resist layer (PMMA 950K 2\% in chlorobenzene) is less sensitive and serves as the actual mask for metal evaporation. Once the resist is spun, a pattern is 'written' by irradiating the PMMA with a beam of electrons, which breaks the bonds in the polymer. The 'exposed' resist is removed from the substrate by immersing the sample in a developer (a 1:3 solution of methyl isobutyl ketone (MIBK) and iso-propyl alcohol (IPA)). Then, the substrate is placed in an e-beam evaporator, where (Cr or Ti)/Pt (5/70 nm) is evaporated. Chromium or titanium is used as a sticking layer for the platinum. We use Pt for the markers because they withstand, without severe deformation, the high temperatures $\left(\sim 900{ }^{\circ} \mathrm{C}\right)$ during nanotube growth. After metal evaporation, the unexposed resist and excess metal are removed by immersing the sample in hot acetone $\left(\sim 55^{\circ} \mathrm{C}\right)$. After lift-off, the substrate contains e-beam markers, AFM markers, as well as a series of optical and numerical markers to help handling and tagging of samples.

We have used two methods to place nanotubes on the substrates: direct deposition from a solution and chemical vapour deposition (CVD) growth. For the first one, we put a small amount of carbon nanotube material in a bottle containing DCE (dichloroethane) and sonicate until the nanotube material has disentangled into separate nanotubes (typically $\sim 30 \mathrm{~min}$ to $1 \mathrm{~h}$ ). Then, a few droplets of solution are placed on a substrate and blown-dried with nitrogen. This process leaves nanotubes all over the substrate. It is easy and fast, but it has certain disadvantages. Many times the nanotubes appear in ropes and not individually. We have also noted that it is harder to make good contact to deposited NTs than to CVD-grown tubes. For these reasons, most of our experiments have been performed with carbon nanotubes grown by CVD. For the catalyst, $40 \mathrm{mg}$ of $\mathrm{Fe}\left(\mathrm{NO}_{3}\right)_{3} \cdot 9 \mathrm{H}_{2} \mathrm{O}$, $2 \mathrm{mg}$ of $\mathrm{MoO}_{2}(\mathrm{acac})_{2}$ and $30 \mathrm{mg}$ of alumina nanoparticles

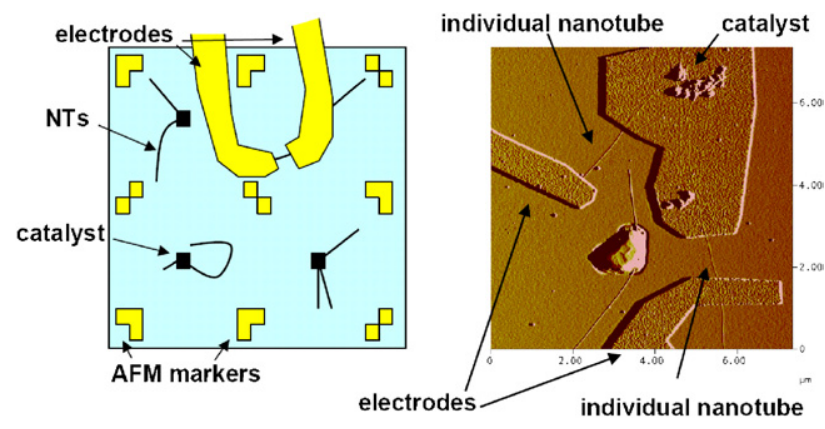

Figure 2. Fabrication process. Left: scheme of a substrate with the AFM markers, catalyst particles at predefined positions, grown nanotubes and designed electrodes. The separation between AFM markers is $6 \mu \mathrm{m}$. Right: actual AFM picture of one of our devices.

are mixed in $30 \mathrm{ml}$ of methanol and sonicated for $\sim 1 \mathrm{~h}$. The resulting liquid catalyst is deposited onto the substrate with $0.5 \mu \mathrm{m}^{2}$ openings in the PMMA resist (patterned on specific known locations by e-beam lithography) and blown dry. After lift-off in acetone, the substrate with patterned catalyst is placed in a 1 inch quartz tube furnace and the CVD is carried out at $900{ }^{\circ} \mathrm{C}$ with $700 \mathrm{sccm} \mathrm{H}_{2}$ and $520 \mathrm{sccm}$ $\mathrm{CH}_{4}$ for $10 \mathrm{~min}$. Argon flows through during heating up and cooling down. The methane and hydrogen flows have been optimized to obtain long and clean nanotubes $(\sim 10 \mu \mathrm{m})$ without amorphous carbon deposition. After growth, typically a few tubes have grown from each catalyst site.

After the nanotube deposition/growth, the substrates are inspected by atomic force microscopy. All our devices have 'customized electrodes', i.e., we design electrodes individually for each nanotube device. We typically choose straight segments of nanotubes located on 'residue-free' areas. The AFM pictures determine the precise location of the nanotubes with respect to the predefined AFM markers. We import these pictures into a CAD program and directly design the electrodes on the top of the desired NTs. A subsequent e-beam lithography step is carried out to write the electrodes and evaporate the metal. The contact metal can be $\mathrm{Cr} / \mathrm{Au}$, $\mathrm{Ti} / \mathrm{Au}, \mathrm{Pd}, \mathrm{Ti} / \mathrm{Al}$, etc depending on the type of experiment. After lift-off, the sample is ready for optical inspection and room temperature characterization. In some cases, we etch part of the $\mathrm{SiO}_{2}$ in order to suspend the nanotubes. This is done by immersing the samples in buffered HF for $\sim 1-2 \mathrm{~min}$, transfer to water and followed by a gentle drying in hot IPA (to prevent the collapse of the nanotube due to surface tension effects).

So far, we have only described the fabrication process for the 'standard' three-terminal nanotube devices. For making more complex device architectures, additional e-beam steps are required. In the following, we describe the extra fabrication steps to make nanotube double quantum dots with tunable tunnel barriers defined by aluminium top gates (see figure 3 ). The tunable tunnel barriers are the very narrow Al top gates. The advantage of a narrow top gate is that it controls the tunnelling barrier on a local scale and only a small portion of the tube is covered with oxide. To fabricate nanotube double dot devices, we first make the Pd contacts to the tubes in the same way as described above. Pd is used, because it introduces little or no barrier at the nanotube-metal contact [14]. In a 


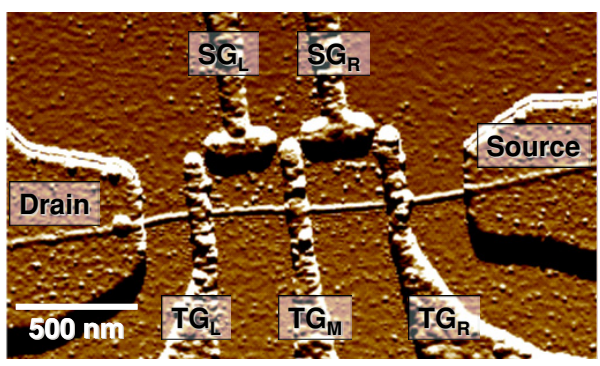

Figure 3. AFM picture of a carbon nanotube double quantum dot device. The contacts (source and drain) are made of $\mathrm{Pd}$ and the gate structures of $\mathrm{Al}$. The top gates $\left(\mathrm{TG}_{\mathrm{L}}, \mathrm{TG}_{\mathrm{M}}\right.$ and $\left.\mathrm{TG}_{\mathrm{R}}\right)$ create tunable tunnel barriers and the side gates are used to change the electrochemical potential of the separate dots individually.

subsequent electron beam lithography step, we make the gate structures. First, we evaporate $2 \mathrm{~nm}$ thin layer of $\mathrm{Al}$ and then this layer is completely oxidized for $10 \mathrm{~min}$ at 1 bar pure oxygen atmosphere. This step can be repeated to obtain a thicker oxide. After the oxidation, evaporation is continued with $35 \mathrm{~nm}$ of $\mathrm{Al}$ and followed by $15 \mathrm{~nm}$ of AuPd.

\section{Semiconducting carbon nanotubes in the few-charge regime}

We focus on the regime of a few charge carriers (electrons or holes) in clean semiconducting nanotubes. High-purity carbon nanotubes (HiPco [15]) are used, which are deposited with low density on a doped Si substrate (serving as a back gate) that has an insulating $\mathrm{SiO}_{2}$ top layer $[16,17]$. Individual nanotubes are electrically contacted with source and drain electrodes (thickness $50 \mathrm{~nm} \mathrm{Au}$ on $5 \mathrm{~nm} \mathrm{Cr}$ ). We then suspend the nanotubes by etching away part of the $\mathrm{SiO}_{2}$ surface [17]. We generally find that removing the nearby oxide reduces the amount of potential fluctuations (i.e. disorder) in the nanotubes, as deduced from transport characteristics.

We focus on a particular semiconducting device that shows regular single QD behaviour for both few-hole and few-electron doping. The distance between the electrodes in this device is $270 \mathrm{~nm}$ (inset in figure $4(a)$ ). The dependence of the linear conductance on gate voltage shown in figure 4(a) is typical for semiconducting p- and n-type behaviour [18]. The conductance in the n-type region is lower than that in the p-type, as usually observed in ambipolar nanotube FETs $[18,19]$. A low-temperature measurement around zero gate voltage (figure $4(b)$ ) shows a large zero-current gap of about $300 \mathrm{meV}$ in bias voltage, reflecting the semiconducting character of this nanotube. The zigzag pattern outside the semiconducting gap is due to Coulomb blockade [20]. These Coulomb blockade features are more evident in figure $4(c)$, where a high-resolution measurement of the differential conductance shows the semiconducting gap with the first two adjacent Coulomb blockade diamonds.

The identification of the Coulomb diamonds for the first electron and first hole allows for an unambiguous determination of the particle number as we continue to fill the QD by further changing the gate voltage. Figure 5(a) shows the filling of holes, one by one, up to 20 holes. The region for the first two holes is enlarged in figure $5(b)$. The
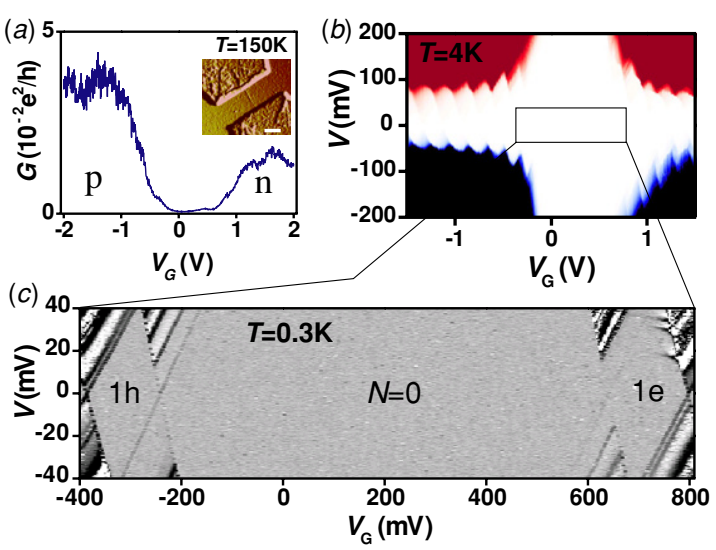

Figure 4. (a) Linear conductance, $G$, as a function of gate voltage, $V_{\mathrm{G}}$, at a temperature $T \sim 150 \mathrm{~K}$ showing the $p$ - and $n$-conducting regions separated by the semiconducting gap. Inset: atomic force microscope image of the device before suspension (scale bar: $200 \mathrm{~nm}$ ). (b) Large-scale plot of the current (in the light regions the current is blocked) versus both $V$ and $V_{\mathrm{G}}$ at $T=4 \mathrm{~K}$. (c) Highresolution measurement of the differential conductance as a function of $V$ and $V_{\mathrm{G}}$ in the central region of $(b)$ at $0.3 \mathrm{~K}$. Between $V_{\mathrm{G}} \sim-250$ and $650 \mathrm{mV}$, the nanotube QD is depleted entirely of mobile charge carriers. As $V_{\mathrm{G}}$ increases (decreases), one electron (hole) enters the dot as indicated in the right (left) Coulomb diamond.

regularity in the Coulomb diamonds indicates a nanotube that is free of disorder. A closer inspection shows that the size of the Coulomb diamonds varies periodically on a smooth background as the hole number increases (figure 5(c)). The alternating, even-odd pattern in this addition energy, $E_{\text {add }}$, reflects the subsequent filling of discrete orbital states with two holes of opposite spin [20]. These features are explained in more detail below.

We first focus on the additional discrete lines outside the Coulomb diamonds running parallel to its edges, as for instance indicated by arrows in figure $5(b)$. Whereas the upper-left edge of the $N$-hole diamond reflects the ground state energy of the $(N+1)$ th hole, the extra lines located at higher voltages, $V$, represent the discrete excitation spectrum for $(N+1)$ holes [20]. The spacing in $V$ directly measures the energy separation between the excitations.

The ambipolar character of the devices allows us to compare the excitation spectra for a particular hole (h) number with the same electron (e) number. The left and right columns in figure 6 show the spectra for, respectively, holes and electrons. The upper row compares the spectra for $1 \mathrm{~h}$ and $1 \mathrm{e}$. The arrows in figure 6(a) point at the first three excited states for a single hole. (Note that only lines with positive slopes are observed because of asymmetric tunnel barriers [20].) The arrows in figure $6(b)$ indicate the corresponding first three excitations for a single electron. Remarkably, we have simply mirror-imaged the arrows from the hole to the electron side without any adjustment of their spacing. We thus find that the $1 \mathrm{~h}$ and 1e excitations occur at the same energy. Since oneparticle systems are free from particle-particle interactions, this symmetry implies that the confinement potential for electrons is the same as for holes.

Electron-hole symmetry also survives interactions as demonstrated in the lower rows in figure 6. Again the arrows 

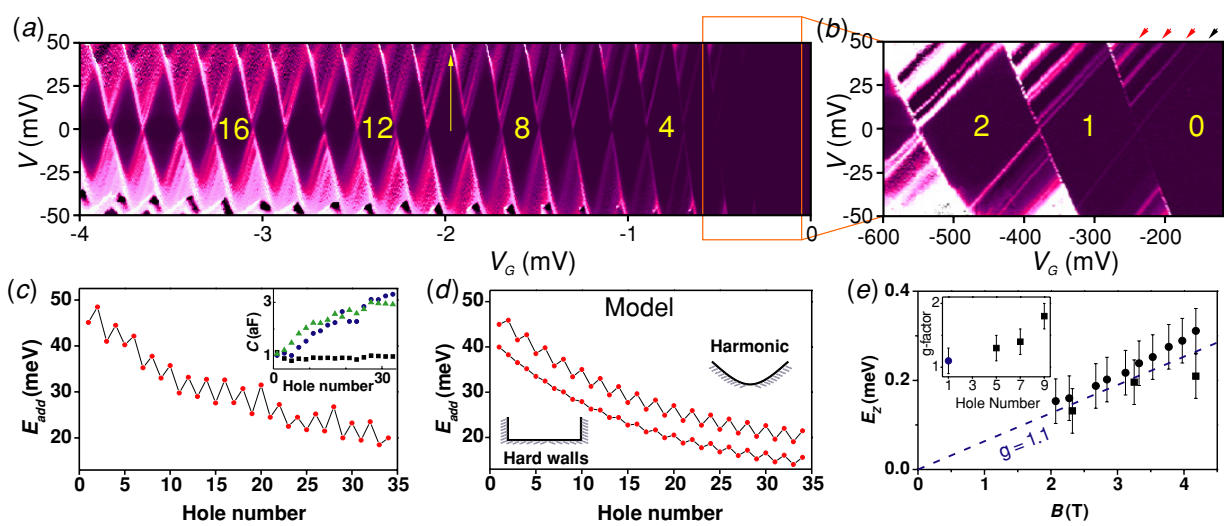

Figure 5. (a) Two-dimensional colour plot of the differential conductance, $\mathrm{d} I / \mathrm{d} V$, versus $V$ and negative $V_{\mathrm{G}}$ at $T=4 \mathrm{~K}$ (black is zero, white is $3 \mu \mathrm{S}$ ). In the black diamond-shaped regions, the number of holes is fixed by the Coulomb blockade. (b) Zoom-in taken at $0.3 \mathrm{~K}$ of the region with 0,1 and 2 holes (white represents $\mathrm{d} I / \mathrm{d} V>10 \mathrm{nS}$ ). Lines outside the diamonds running parallel to the edges correspond to discrete energy excitations (the black arrow points at the one-electron ground state and the light (red) arrows at the one-electron excited states). (c) Addition energy, $E_{\text {add }}$, as a function of hole number. $E_{\text {add }}$ is deduced from the diamond size for positive and negative $V$. Inset: the capacitances $C_{\mathrm{S}}$ (green), $C_{\mathrm{D}}$ (blue) and $C_{\mathrm{G}}$ (black) versus hole number. $(d)$ Calculation of the addition energy spectrum for a semiconducting nanotube (as an example we have taken a zigzag $(35,0)$, with $E_{\text {gap }} \sim 259 \mathrm{meV}, m_{\text {eff }}=0.037 m_{\mathrm{e}}$ ) for a harmonic potential (top) and a hard-wall potential (bottom). The parameters for the harmonic potential are $V(x=135 \mathrm{~nm})=E_{\text {gap }} / 2$. (e) Zeeman splitting energy, $E_{\mathrm{Z}}$, versus magnetic field, $B$, for the one-hole orbital states. Inset: $g$-factor as a function of hole number.
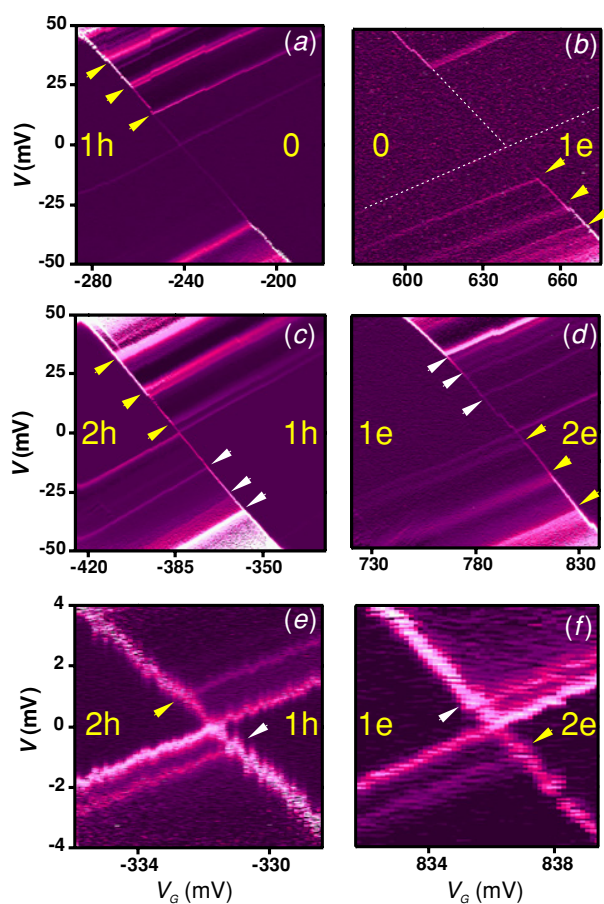

Figure 6. Excitation spectra for different electron and hole numbers demonstrating electron-hole symmetry. $\mathrm{d} I / \mathrm{d} V$ is plotted versus $\left(V, V_{\mathrm{G}}\right)$ at $T=0.3 \mathrm{~K}$. (a) The transition from the 0 to $1 \mathrm{~h}$ Coulomb diamonds. (b) Corresponding transition from 0 to $1 \mathrm{e}$. The white dotted lines in $(b)$ are guides to the eye to indicate the diamond edge (not visible for this choice of contrast). (c) and (d) Same for 1-2h and 1-2e. $(e)$ and $(f)$ Low-bias zoom-in of the 1-2h and 1-2e crossings.

pointing at the hole excitations have simply been mirrorimaged to the electron side. Thus, we find that the spectra for $2 \mathrm{~h}$ and $2 \mathrm{e}$ and for $3 \mathrm{~h}$ and $3 \mathrm{e}$ (not shown here) show virtually perfect electron-hole symmetry in the excitation spectra. From a closer look, one can see that also the relative intensities of the excitation lines display electron-hole symmetry.

The quality of our data allows for a quantitative analysis. The addition energy is defined as the change in electrochemical potential when adding the $(N+1)$ charge to a QD already containing $N$ charges. The constant-interaction (CI) model [20] gives $E_{\text {add }}=U+\delta E$, where $U=e^{2} / C$ is the charging energy $\left(C=C_{\mathrm{S}}+C_{\mathrm{D}}+C_{\mathrm{G}}\right)$ and $\delta E$ is the orbital energy difference between $N+1$ and $N$ particles on the QD. In the case of a semiconductor QD, the addition energy for adding the first electron to the conduction band equals $U+E_{\text {gap }}$. From the observed gap size of $300 \mathrm{meV}$ and $U \sim 50 \mathrm{meV}$, we determine the semiconducting gap $E_{\text {gap }} \sim 250 \mathrm{meV}$, which corresponds to a nanotube diameter of $2.7 \mathrm{~nm}$ [21]. AFM measurements, that usually underestimate the real height [22], indicate an apparent tube height of $1.7 \pm 0.5 \mathrm{~nm}$.

Since two electrons with opposite spin can occupy a single orbital state, the CI model predicts an alternating value for $E_{\text {add }}$, where $E_{\text {add }}=U$ for $N=$ odd and $E_{\text {add }}=U+\delta E$ for $N=$ even. We indeed observe such an even-odd alternation in figure 5(c) with $\delta E \sim 4.3 \mathrm{meV}$ throughout the entire range of $N=1$ to $N=30$. Measurements of the Zeeman spin splitting in a magnetic field [23] confirm our assignment of even-odd particle number: lines corresponding to ground states for odd $N$ split (i.e. total $\operatorname{spin}=1 / 2$ ), whereas even- $N$ lines do not split (i.e. total spin $=0$ ). The data in the lower inset of figure 5(c) yield a $g$-factor, $g=1.0 \pm 0.2$, which is a factor of 2 lower than that reported in metallic nanotubes [3,24]. Lower $g$-factors are usually due to spin-orbit coupling, but this effect is small for carbon. It may hint at strong electron-electron interactions in the 1D QD.

The addition energy spectrum indicates that $\delta E \approx$ $4.3 \mathrm{meV}$ for consecutive states as we fill the QD with holes. Previous spectra from metallic nanotubes have been analysed by considering a hard-wall potential in the nanotube, with an effective mass determined by the band structure. Our data show that this approach is not justified for semiconducting 

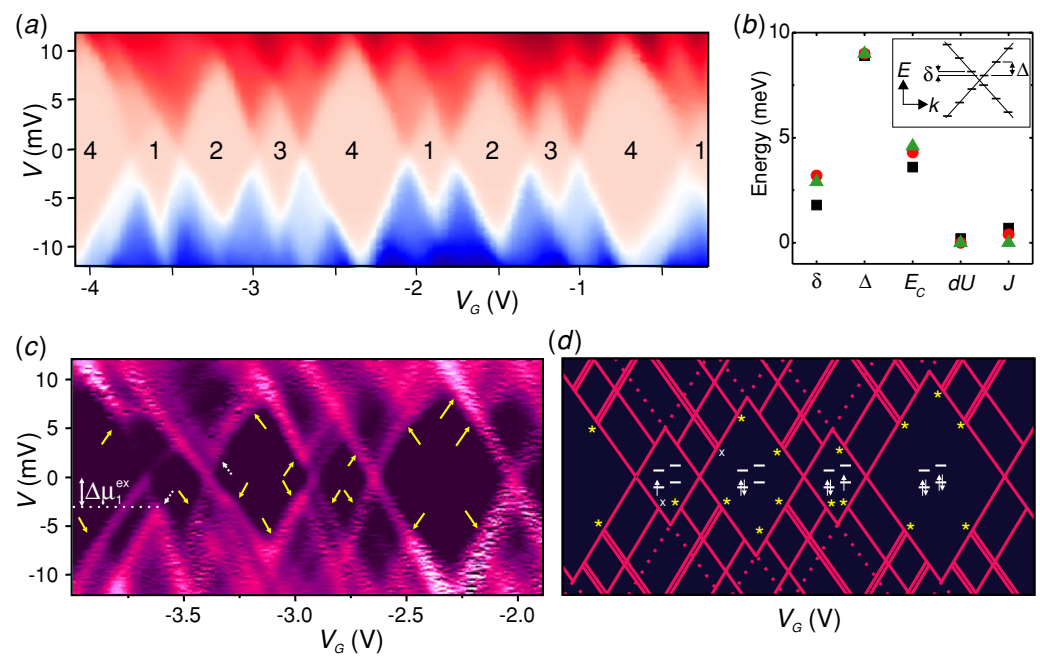

Figure 7. (a) Current as a function of $V$ and $V_{\mathrm{G}}$ at $T=300 \mathrm{mK}$. Current ranges from $-40 \mathrm{nA}$ to $+40 \mathrm{nA}$. (b) Values of the parameters for three different groups of four. In the inset, the low-energy band structure of a metallic SWNT is shown. For a finite-length SWNT, the wave vector $k$ is quantized along the tube axis which leads to a set of quantized energy levels separated by $\Delta$ in each band. $\delta$ is the mismatch between the two bands. $(c)$ The differential conductance $(\mathrm{d} I / \mathrm{d} V)$ for the first group from $(a)$. Black is zero and bright is $>12 \mu \mathrm{S}$. Lines running parallel to the diamond edges correspond to discrete energy excitations. The excitation energies corresponding to the dashed arrows have been used to deduce the model parameters. The predicted excitations are indicated by (yellow) arrows. $(d)$ Calculated spectrum. The stars correspond to the arrows in $(c)$. The white diagrams indicate the spin filling of the ground state.

nanotubes. Lack of effective screening in 1D and the low number of mobile charges yield a gradual potential decay from the contacts [25]. We have computed the addition energy spectrum for a semiconducting nanotube whose gap is $\sim 250 \mathrm{meV}$ for two situations (figure $5(d)$ ): hard-wall and harmonic potentials of height $E_{\text {gap }} / 2$ at the contacts [25]. For hard walls, the level spacing increases slowly up to $\sim 1.9 \mathrm{meV}$ for $N=34$. In the case of a harmonic potential, the level spacing is constant, as in the experiment, and equals $2.7 \mathrm{meV}$, in reasonable agreement with the experimental value of $\sim 4.3 \mathrm{meV}$.

On the top of the predicted even-odd pattern, there is a monotonic decrease of the average charging energy with $N$, implying that the total capacitance is changing. We have performed a detailed analysis of the QD electrostatics following [26]. The result is given in the top-right inset of figure 5(c). It shows that the change in $C$ is mainly due to an increase in $C_{\mathrm{S}}$ and $C_{\mathrm{D}}$. This increase can be assigned to a decrease of the tunnel barrier widths as $\left|V_{\mathrm{G}}\right|$ increases, consistent with the simultaneous decrease of $\mathrm{d} I / \mathrm{d} V$ in figure $5(b)$. Indeed, $\mathrm{d} I / \mathrm{d} V$ varies from $5 \mathrm{G} \Omega$ in the first Coulomb peak to $400 \mathrm{k} \Omega$ at large negative $V_{\mathrm{G}}$.

The observation of electron-hole symmetry imposes severe restrictions on the QD system: the effective masses for holes and electrons should be equal and the QD should be free of disorder. Scattering by negatively charged impurities, for example, is repulsive for electrons but attractive for holes, so it would break electron-hole symmetry. A symmetric band structure has been theoretically predicted for graphite materials and carbon nanotubes [21].

A detailed analysis of the excitation spectrum requires calculations that are beyond the constant-interaction model. The change in orbital energy when adding a charge is unambiguously given by $\Delta E \sim 4.3 \mathrm{meV}$, seemingly independent of $N$. Excitations of a smaller energy scale have to be related to interactions. The likely interactions in semiconducting nanotubes are the following. (1) Exchange interaction between spins (e.g. spin $=1$ triplet states gain energy from the exchange interaction). Note that we observe an even-odd pattern, which seems to exclude ground states with spins $>1 / 2$. Excited states, however, can have spins $>1 / 2$. (2) Electron-phonon interactions. The vibrational modes in a suspended nanotube also have a discrete spectrum, which can show up in the excitation spectra [27]. Note that vibrational modes do not affect the addition spectrum of the ground states. (3) Electron-electron interactions. The value for the interaction strength parameter $U / \Delta E \sim 10$. Such a large $U / \Delta E$ ratio points to the presence of phenomena that are not captured by the CI model. Luttinger liquid models developed for finite-length metallic nanotubes are not applicable to our few-particle nanotubes. A more appropriate starting point is the exact calculations for 1D QDs. In the few-particle regime, the charge carriers tend to localize and maximize their separation, thereby forming a Wigner crystal [28]. In such a Wigner state, the spectrum consists of both high-energy single-particle excitations and collective excitations at low energy [29], similar to our experiment.

\section{Excitation spectrum and shell filling in metallic carbon nanotubes}

The two-fold degenerate, low-energy band structure of a metallic SWNT is schematically shown in the inset of figure $7(b)$. Quantization along the nanotube axis leads to a set of single-particle states that are equally spaced because of the linear energy dispersion relation [21]. The combination of the two bands and the spin yields a four-fold periodicity in the electron addition energy. This four-fold periodicity has been measured by different groups [30-34]. To describe CNT QDs, the CI model has been extended [35] to include five 
independent parameters: the charging energy $E_{\mathrm{C}}$, the quantum energy level separation $\Delta$, the subband mismatch $\delta$ (see inset in figure $7(b)$ ), the exchange energy $J$ and the excess Coulomb energy $\mathrm{d} U$ (the excess interaction of two electrons occupying the same level). The CNT QD has an energy gain of $J$ when two electrons have aligned spins compared to the situation of two electrons having opposite spins. An independent verification of this extended CI model [35] requires the observation of the ground-state addition energies and of at least two excited states. In this section, we investigate the excitation spectrum of closed SWNT QDs. Not only the ground but also the complete excited state spectrum of these QDs has been measured by transport spectroscopy experiments, enabling us to determine all five parameters independently. With these, the remaining measured excitation energies are well predicted leading to a complete understanding of the spectrum, without adjustable parameters.

The device is made from a HiPco NT [15] with $L=$ $180 \mathrm{~nm}$ and a diameter of $1.1 \mathrm{~nm}$ as determined by AFM. It is contacted by evaporating $\mathrm{Cr} / \mathrm{Au}(5 / 75 \mathrm{~nm})$ electrodes. Figure 7(a) shows the current, $I$, as a function of source-drain bias voltage, $V$, and gate voltage, $V_{\mathrm{G}}$. In the light-coloured diamond-shaped regions, the current is blocked due to $\mathrm{CB}$ and the number of electrons is fixed. The clear four-fold periodicity makes it possible to assign the number of electrons in the last occupied shell. The sizes of the diamonds form an interesting pattern, namely a repetition of small medium small big. This pattern is a consequence of the large subband mismatch compared to the exchange energy, as we discuss below. The addition energy is obtained by multiplying the diamond width, $\Delta V_{\mathrm{G}}$, by a conversion factor, $\alpha(\approx 0.017)$, which relates the gate voltage scale to the electrochemical potential [26].

The Oreg et al model [35] yields the following equations for the addition energy of the $N$ th electron added ${ }^{1}$ :

$$
\begin{gathered}
\Delta \mu_{1}=\Delta \mu_{3}=E_{\mathrm{C}}+\mathrm{d} U+J, \\
\Delta \mu_{2}=E_{\mathrm{C}}+\delta-\mathrm{d} U, \\
\Delta \mu_{4}=E_{\mathrm{C}}+\Delta-\delta-\mathrm{d} U .
\end{gathered}
$$

To extract all five parameters, two more equations are needed. These are provided by the excitation spectrum. In figure 7(c), we show the numerical derivative of figure $7(a)$ (i.e., the differential conductance) for the first group of four. Excited states of the electrons are visible for all diamonds. The value of a particular excitation energy equals the bias voltage at the intersection between the excitation line and the Coulomb diamond edge (see figure $7(c)$ ). The dotted (white) arrows in diamonds 1 and 2 in figure $7(c)$ correspond to the first excitation for one and two electrons extra on the CNT QD, respectively. The theoretical values of these two energies are

$$
\begin{gathered}
\Delta \mu_{1}^{\mathrm{ex}}=\delta, \\
\Delta \mu_{2}^{\mathrm{ex}}=\delta-J-\mathrm{d} U .
\end{gathered}
$$

Equations (1)-(5) allow us to uniquely determine the five unknown parameters from the experimental data alone. We find $E_{\mathrm{C}}=4.3 \mathrm{meV}, \Delta=9.0 \mathrm{meV}, \delta=3.2 \mathrm{meV}, J=0.4 \mathrm{meV}$ and $\mathrm{d} U \approx 0 \mathrm{meV}$. The values of the parameters do not vary

1 This set of equations corresponds to a singlet ground state for $N=2$. The triplet case is incompatible with the experimental data.
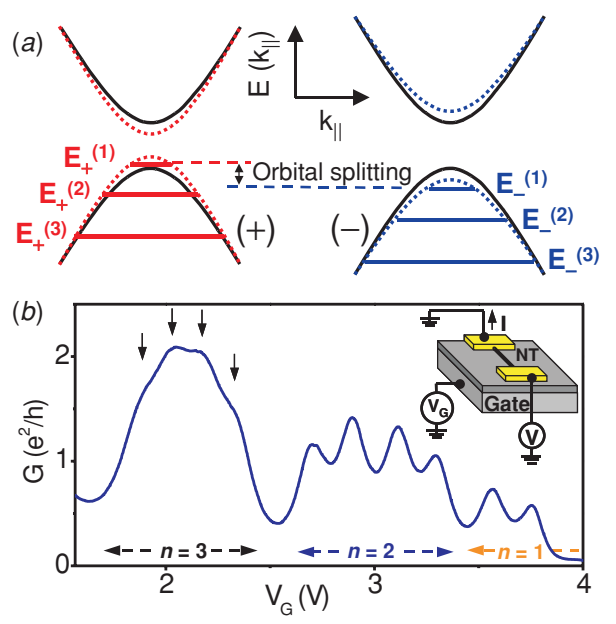

Figure 8. (a) Schematic band structure of a CNT near its energy gap. Black lines represent the one-dimensional energy dispersion relation, $E\left(k_{\|}\right)$, at $B=0\left(k_{\|}\right.$is the wave vector along the CNT axis). The valence (conduction) band has two degenerate maxima (minima). Size quantization in a finite-length CNT results in a set of discrete levels with both spin and orbital degeneracy. The degeneracy is lifted by a magnetic field parallel to the CNT. The 1D subbands (and the corresponding levels) at finite $B$ are represented by (red and blue) dotted (solid) lines. Only the orbital splitting of the energy levels is shown in this figure. (b) Linear conductance, $G$, versus gate voltage, $V_{\mathrm{G}}$, taken at $T=8 \mathrm{~K}$. Inset: device scheme.

significantly between the different groups of 4 , as shown in figure $7(b)$. The theoretically expected value for the level spacing is $\Delta=h v_{\mathrm{F}} / 2 L$ [3]. With $v_{\mathrm{F}}=8.1 \times 10^{5} \mathrm{~m} \mathrm{~s}^{-1}$ [36] and $L=180 \mathrm{~nm}$ (the CNT length between the contacts), we find $9.3 \mathrm{meV}$ in excellent agreement with the experimental value.

Figure $7(d)$ shows the calculated spectrum of the NT QD using the parameters deduced from the experiment. Some excitations are split by the exchange energy. The stars in the calculated spectrum correspond to the arrows in the experimental data. The excitations denoted with ' $x$ ' were used for obtaining the parameters and correspond to the dashed arrows in figure $7(c)$. The calculated spectrum resembles the measured one strikingly well. Although the results in this section have been obtained in a HiPCO tube, four-fold shell filling is also commonly observed in our CVD grown nanotubes.

\section{Magnetic field dependence of carbon nanotube quantum dot excitations exhibiting a four-fold shell structure}

In this section, we describe magnetic field dependent electronic transport spectroscopy measurements on CNT QDs exhibiting a four-fold shell structure. We show that (i) each shell consists of two orbitals with opposite magnetic moment, (ii) the splitting of the orbital states with $B$ accounts for all the observed transitions in the spin and orbital configuration of the CNT QD, (iii) a weak coupling exists between orbitals with opposite magnetic moment resulting in level repulsion at $B=0$, and (iv) Zeeman and orbital contributions to the electron magnetic moment can be distinguished by inelastic co-tunnelling spectroscopy. 
Ajiki and Ando [37] predicted that the orbital degeneracy should be lifted by a magnetic field parallel to the CNT axis (figure $8(a)$ ). This effect can be understood by noting that, due to clockwise and anti-clockwise motion around the tube, electrons in degenerate ' + ' and ' - ' subbands should have opposite orbital magnetic moments, $\mu_{\text {orb }}$. In the case of finitelength CNTs, a discrete energy spectrum is expected from size quantization. The level spectrum of a CNT QD can then be described as two sets of spin-degenerate levels, $E_{+}^{(n)}$ and $E_{-}^{(n)}$, where $n=1,2,3, \ldots$ is the quantum number in the longitudinal direction (see figure $8(a)$ ). In the absence of intersubband mixing, $E_{+}^{(n)}=E_{-}^{(n)}$ at $B=0$, a four-fold degenerate shell structure is expected for every $n$. Below we show that a finite coupling can exist, resulting in a small orbital splitting even at $B=0$.

The four-fold shell filling emerges in a measurement of the linear conductance, $G$, versus gate voltage, $V_{G}$. This is shown in figure $8(b)$ for a QD device fabricated from a metallic nanotube with a small band gap ${ }^{2}$ [38]. $G$ exhibits Coulomb blockade oscillations [26, 20] corresponding to the filling of the 'valence' band of the CNT. From left to right, electrons are consecutively added to the last three electronic shells, $n=3$, 2 and 1, respectively. The shell structure is apparent from the $V_{\mathrm{G}}$ spacing between the Coulomb oscillations. The addition of an electron to a higher shell requires an extra energy cost corresponding to the energy spacing between shells. This translates into a larger width of the Coulomb valley associated with a full shell ${ }^{3}$. The first group of four Coulomb peaks on the left-hand side of figure $8(b)(n=3)$ is strongly overlapped due to a large tunnel coupling to the leads and the Kondo effect [8]. The coupling decreases with $V_{\mathrm{G}}$ and becomes very small near the band gap, which lies just beyond the right-hand side of the $V_{\mathrm{G}}$ range shown. Due to this small coupling, the Coulomb peaks associated with the last two electrons in $n=1$ are not visible (see figure $9(b)$ ).

The shell structure breaks up at finite $B$ (figure $9(a)$ ), which shows gate traces as a function of magnetic field. Peaks in the conductance appear white. In each group of four Coulomb peaks, the first (last) two peaks shift towards lower (higher) $V_{\mathrm{G}}$. This behaviour demonstrates the strong $B$-dependence of the orbital levels described in figure $8(a)$. The magnetic field shifts the '-' orbital levels down in energy, while the ' + ' orbitals are shifted up due to their opposite $\mu_{\text {orb }}$. Consequently, the addition of the first (last) two electrons to a shell results in a pair of Coulomb peaks shifting towards lower (higher) $V_{\mathrm{G}}$. For each shell, $\mu_{\mathrm{orb}}$ can be extracted from the shift, $\Delta V_{\mathrm{G}}(n)$, in the position of the corresponding Coulomb peaks. Neglecting the Zeeman splitting, we use the relation $e \alpha \Delta V_{\mathrm{G}}(n)=\left|\mu_{\mathrm{orb}}(n) \cos \varphi \Delta B\right|$, where $\Delta B$ is the change in $B, \varphi$ is the angle between the nanotube and $B$, and $\alpha$ is the capacitance ratio extracted from nonlinear measurements. The values obtained $\left(0.90,0.80\right.$ and $0.88 \mathrm{meV} \mathrm{T}^{-1}$ for $n=1$, 2 and 3 , respectively) are an order of magnitude larger than the electron spin magnetic moment $\left(1 / 2 g \mu_{\mathrm{B}}=0.058 \mathrm{meV} \mathrm{T}^{-1}\right.$

2 This band gap can be due to perturbations such as curvature or strain. The measured value of the band gap is $\sim 30 \mathrm{meV}$.

3 From measurements in the nonlinear regime (not shown), we estimate $\Delta_{1,2} \sim 3 \mathrm{meV}$ and $\Delta_{2,3} \sim 5 \mathrm{meV}$, where $\Delta_{n, n+1} \equiv E_{+}^{(n)}-E_{+}^{(n+1)}=$ $E_{-}^{(n)}-E_{-}^{(n+1)}$. The Coulomb charging energy is $U \sim 5 \mathrm{meV}$.

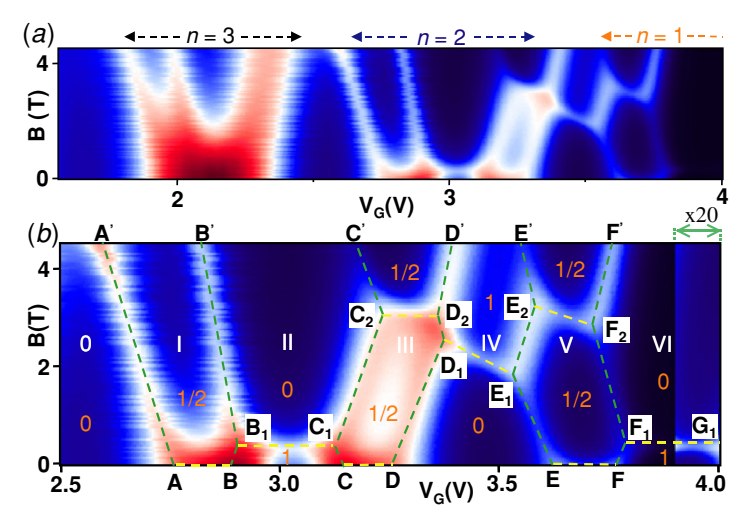

Figure 9. $G$ versus $B$ on a colour scale at $T=0.34 \mathrm{~K}$ for the $V_{\mathrm{G}}$ range shown in figure $8(b)$. Red, white and blue indicate high, intermediate and low $G$, respectively. The conductance range is 0 (dark blue) to $3 e^{2} / h$ (dark red). (b) Zoom-in of $(a)$. Here $G$ ranges from 0 (dark blue) to $2 e^{2} / h$ (dark red). The green dashed lines highlight the evolution of the Coulomb peaks with $B$. They are labelled as $\mathrm{AA}^{\prime}, \mathrm{BB}^{\prime}, \ldots, \mathrm{FF}^{\prime}$. These divide the plot into different Coulomb blockade regions indicated by the number of electrons in the last two shells (white numbers 0 to VI). The high- $G$ regions (indicated by yellow dashed lines) in between Coulomb peaks are due to the Kondo effect. Numbers (orange) indicate the spin in each region. On the right-hand side, $G$ is multiplied by 20 , so that the triplet-singlet transition is clearly seen along $\mathrm{F}_{1} \mathrm{G}_{1}$.

for $g=2$ ) and in good agreement with an estimate of $\mu_{\text {orb }}$ based on the nanotube diameter ${ }^{4}$.

The strong $B$-dependence of the orbital states induces changes in the orbital and spin configuration of the QD similar to previous findings in semiconducting QDs [39]. These are reflected as kinks in the evolution of the Coulomb peaks with $B$ (figure $9(b)$ ). Remarkably, a fully consistent description of the $B$-dependent energy spectrum and the ground-state spin/orbital configuration can be obtained through a careful analysis of all the kinks in figure $9(b)$ [40].

Note that kinks in figure $9(b)$ are connected by conductance ridges crossing the Coulomb valleys. The enhancement of $G$ at these ridges is due to Kondo effects of different origins. At $\mathrm{B}_{1} \mathrm{C}_{1}, \mathrm{D}_{1} \mathrm{E}_{1}$ and $\mathrm{F}_{1} \mathrm{G}_{1}$, the Kondo effect arises from singlet-triplet degeneracy [41]. At AB, CD and $\mathrm{EF}$, an enhanced Kondo effect is observed in relation to orbital degeneracy [8]. The Kondo ridges at $\mathrm{C}_{2} \mathrm{D}_{2}$ and $\mathrm{E}_{2} \mathrm{~F}_{2}$ are due to the recovery of orbital degeneracy between $E_{-}^{(2)}$ and $E_{+}^{(1)}$ [8]. Note that, as a result of electron-hole symmetry, region III (three electrons in shell $n=2$ ) and region V (one electron in shell $n=1$ ) have a certain degree of mirror symmetry, both in terms of the slope of the Coulomb peaks' evolution with $B$ and the Kondo ridges.

The data shown so far have been explained in terms of a $B$-induced splitting of orbital degeneracy, as if the two orbital states of every shell were degenerate at $B=0$. A small zerofield orbital splitting, however, may exist and it may be masked by the Kondo effect at $\mathrm{AB}, \mathrm{CD}$ and $\mathrm{EF}$. In order to investigate this possibility, we considered a different device, which has a much smaller coupling to the leads and hence a much weaker Kondo effect. This device also exhibits a four-fold

4 From the value for $n=1$, we extract a nanotube diameter $D=4 \mu_{\mathrm{orb}} / e v_{\mathrm{F}}=$ $4.5 \mathrm{~nm}$, in agreement with the measured diameter of $4.0 \pm 0.5 \mathrm{~nm}$ as determined by atomic force microscopy. For this device, $\varphi=37^{\circ}$. 

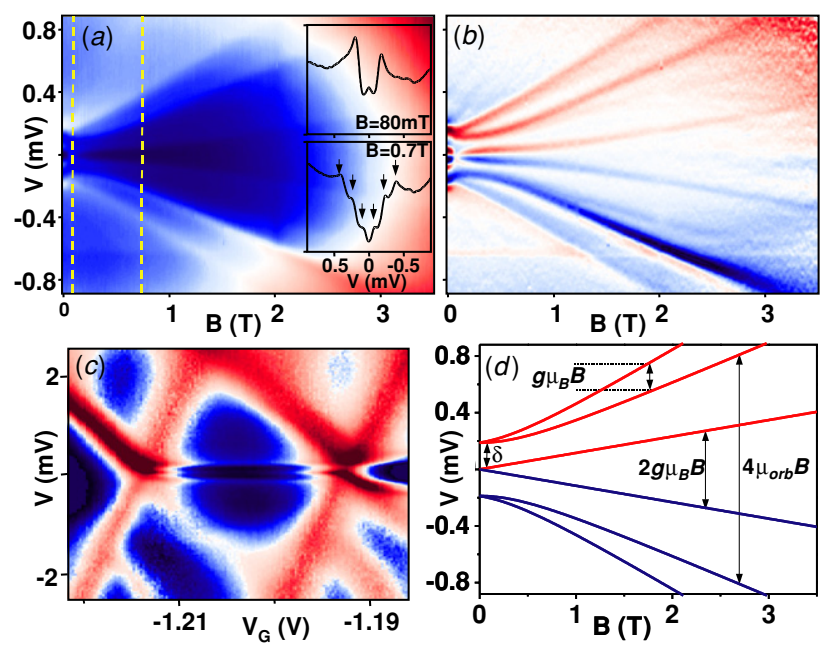

Figure 10. (a) Colour plot of the differential conductance, $\mathrm{d} I / \mathrm{d} V$, versus bias, $V$, and $B$, measured in the centre of the Coulomb diamond (see $(c))$ at $T=30 \mathrm{mK}$. The (yellow) dashed lines indicate the traces shown in the top and bottom insets. Insets: (top) $\mathrm{d} I / \mathrm{d} V$ trace taken at $B=80 \mathrm{mT}$, showing the onset of inter-orbital IC and a small zero bias peak due to ordinary spin- $1 / 2$ Kondo effect. The vertical axis scale spans from 0.02 to $0.08 e^{2} / h$; (bottom) same as the top inset, but at $B=0.7 \mathrm{~T}$, showing the six IC steps.

(b) Numerical derivative of the $\mathrm{d} I / \mathrm{d} V$ plot in $(a)$. The two inner lines result from Zeeman splitting of the Kondo peak at $B=0$. The outer lines represent the $B$-evolution of the spin-split orbital levels. (c) $\mathrm{d} I / \mathrm{d} V$ versus $V$ and $V_{\mathrm{G}}$, for a single electron in a shell at $B=80 \mathrm{mT}$. $(d)$ Calculated $B$-dependence of the IC spectrum for a single electron in a spin-degenerate level for two coupled orbitals. Red (blue) lines indicate upwards (downwards) steps in $\mathrm{d} I / \mathrm{d} V$ with increasing $V$.

periodicity in the Coulomb peaks' pattern. Figure 10(c) shows a Coulomb diamond corresponding to one electron in a shell at $B=80 \mathrm{mT}$. Inside the diamond, single-electron tunnelling is suppressed and transport occurs via higher order co-tunnelling processes. A sharp increase in the differential conductance, $\mathrm{d} I / \mathrm{d} V$, is observed at a bias $|V|=V_{\text {in }} \sim 190 \mu \mathrm{V}$, denoting the onset of inelastic co-tunnelling (IC) [26, 42, 43]. The IC transition takes place between the two spin-degenerate orbital levels of the same shell (see the discussion below), thereby indicating the existence of a finite splitting at $B=0$. Before discussing the $B$-dependence of the IC edges, we note that a weak Kondo peak is also present at $V=0$ (top inset in figure $10(a)$ ). This Kondo effect arises from the single-electron occupancy of the spin-degenerate orbital ground state.

At finite $B$, both the Kondo peak and the IC edges split due to Zeeman spin splitting. This is shown in figure 10(a), where $\mathrm{d} I / \mathrm{d} V$ is plotted versus $(V, B)$ for $V_{\mathrm{G}}$ at the centre of the Coulomb diamond. In order to identify the $\mathrm{d} I / \mathrm{d} V$ steps more clearly, figure $10(b)$ shows the numerical derivative of the $\mathrm{d} I / \mathrm{d} V$ plot in figure $10(a)$ (i.e. $\mathrm{d}^{2} I / \mathrm{d} V^{2}$ versus $V$ and $\left.B\right)$. IC steps in figure $10(a)$ turn into peaks $(V>0)$ or dips $(V<0)$ in figure $10(b)$. The zero-bias Kondo peak evolves into two $\mathrm{d} I / \mathrm{d} V$ steps at $V=g \mu_{\mathrm{B}} B / e(g=2)$. These correspond to IC processes in which the spin state of the QD is flipped, i.e. from $|-, \uparrow\rangle$ (ground state) to $|-, \downarrow\rangle$ (excited state). Each of the two $\mathrm{d} I / \mathrm{d} V$ steps associated with inter-orbital IC splits by $g \mu_{\mathrm{B}} B / e$ and they move further apart due the increasing orbital splitting, $2 \mu_{\mathrm{orb}} B \cos \varphi\left(\varphi=33^{\circ}\right)$, as illustrated in figure $10((d))$. We estimate $2 \mu_{\mathrm{orb}} \sim 350 \mu \mathrm{eV} \mathrm{T} \mathrm{T}^{-1}$, i.e. $\sim 3$ times the Zeeman splitting. The six steps ('Zeeman', 'orbital' and 'orbital + Zeeman') can be seen in the bottom inset of figure 10(a). An important consequence of the angular dependence of the orbital splitting is that researchers have now two 'semi-independent' knobs to control the energy spectrum of CNT QDs ( $B$ controls the Zeeman splitting and the angle nanotube $-B$ controls the orbital splitting).

The evolution of the outer IC peaks is nonlinear at low $B$, indicating an anti-crossing between the ' + ' and '- ' orbital levels. Such an IC spectrum can be readily modelled using a Hamiltonian that includes an inter-orbital coupling besides orbital and Zeeman terms. The corresponding energy eigenstates are $E= \pm \sqrt{(\delta / 2)^{2}+\left(\mu_{\text {orb }} B \cos \varphi\right)^{2}} \pm 1 / 2 g \mu_{\mathrm{B}} B$ (the four possible sign combinations). The IC spectrum calculated with this simple model (figure $10(d)$ ) clearly accounts for the experimental data. The nonlinear evolution of the orbital splitting with $B$ constitutes direct evidence that the so-called subband level mismatch, usually denoted by $\delta$ [30-35], is due to a small, but finite, quantum-mechanical coupling between the two orbital subbands in carbon nanotubes.

\section{Excitations in suspended carbon nanotube quantum dots}

So far, we have considered excitations arising from the electronic structure of the NT. In this section, we investigate suspended CNT QDs to probe the effect of vibrations on electron transport. We have performed low-temperature electronic transport spectroscopy measurements on suspended single-wall NTs of different lengths [44]; here we focus on a single device. In figure $11(a)$, we show a stability diagram $[20,26]$ for a suspended NT sample, with a length of 1.2 $\mu \mathrm{m}$ and a diameter of about $1 \mathrm{~nm}$, measured at $10 \mathrm{mK}$. The differential conductance, $\mathrm{d} I / \mathrm{d} V$, is plotted as a function of bias and gate voltages. In the diamond-shaped regions (Coulomb diamonds), the current is blocked due to the Coulomb blockade and the charge number in the dot is fixed. Regular and closing Coulomb diamonds indicate single dot behaviour [20]. For the gate voltage range shown, single dot behaviour is observed. The inset in figure 11(a), which was taken at a higher temperature $(300 \mathrm{mK})$ in a different cool down, illustrates that the diamonds close. The low bias current, however, is suppressed which could be a signature of strong electron-phonon coupling [45-47].

As shown in section 3, electronic excitations in nanotubes typically differ between adjacent charge states [24, 32]. In figure 11(a), a dense set of equally spaced excitation lines (starting from the first electronic excitation) is clearly visible near $V_{\mathrm{G}}=210 \mathrm{mV}$ and $230 \mathrm{mV}$, i.e., adjacent charge states exhibit a similar set of excitations with approximately the same energy spacing. The fact that excitations occur primarily in one direction is due to asymmetric tunnel barriers [20]. The energy difference between the excitation lines in figure 11( $a$ ) is shown in figure $11(c)$. Clearly, the excitation energy is an integer multiple of the first (fundamental) excitation. Thus, they form a harmonic spectrum with up to five levels. A linear fit yields an excitation energy of $140 \mu \mathrm{eV}$ for this $1.2 \mu \mathrm{m}$ long 


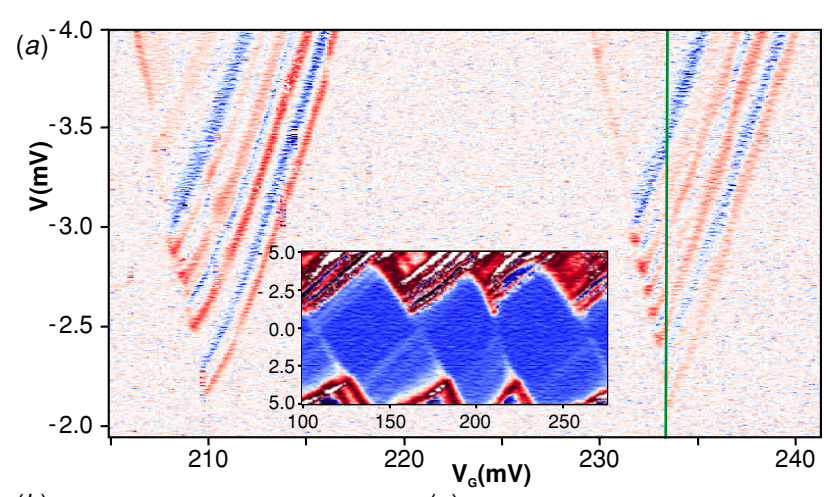

(b)
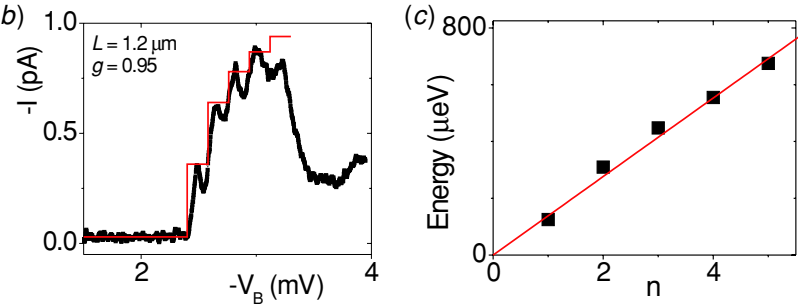

Figure 11. (a) Stability diagram for a suspended nanotube with $1.2 \mu \mathrm{m}$ length and a diameter of about $1 \mathrm{~nm}$. The conductance $(\mathrm{d} I / \mathrm{d} V)$ is plotted as a function of source-drain voltage, $V$, and gate voltage, $V_{\mathrm{G}}$. The range is from low to high conductance (blue to red). Measurement has been done at $T=10 \mathrm{mK}$ base temperature. In the inset, a measurement performed at $300 \mathrm{mK}$ temperature with regular closing Coulomb diamonds is shown to illustrate that we have single quantum dot behaviour for the shown gate voltage range. In the main figure, a strong suppression of the conductance for low bias is present. We furthermore observe a closely spaced set of lines running parallel to the Coulomb diamond edges for two charge states. These lines are excitations of the quantum dot. (b) The current-voltage characteristic taken along the (green) line in (a). The (red) lines represent the step heights calculated in the Franck-Condon model (see the text) for an electron-phonon coupling of 0.95 . The calculated step heights follow the measured steps well. (c) The energy separation between the excitation lines is plotted. Clearly, the separation is equidistant and thus we have a harmonic spectrum. The slope of the linear fit is $140 \mu \mathrm{eV}$.

nanotube. This value is an order of magnitude smaller than the mean electronic level spacing given by $\Delta=h v_{\mathrm{F}} / 2 L$.

The observed harmonic spectra indicate the presence of a vibrational mode coupled to electron tunnelling [27]. Multiple steps with identical spacing would then arise from the excitation of an integer number of vibrational quanta. Indeed, the observed equidistant energy separation is consistent with that expected from the longitudinal stretching mode in the nanotubes. In figure 12, we plot the energy of important lowenergy vibrational modes of single-wall nanotubes [50-52]. For comparison, we plot the mean electronic energy level separation, $\Delta$, in black. Squares (blue) correspond to the fundamental vibrational excitation energy extracted from the linear fit in figure 11(c) and for two other devices [44]. The energy of the radial breathing mode (green) does not depend on the nanotube length and equals $28 \mathrm{meV} / d(\mathrm{~nm})$ [50]. The bending mode (red) has a $L^{-2}$ dependence [53] and an energy much smaller than the measured excitation energy. The stretching mode vibration energy (blue) is inversely proportional to the length [54], $E=(n h / L) \sqrt{Y / \rho_{\mathrm{m}}}$, where $Y$ is Young's modulus, $\rho_{\mathrm{m}}$ is the density and $n$ is the vibrational quantum number. For nanotubes with $\rho_{\mathrm{m}}=$

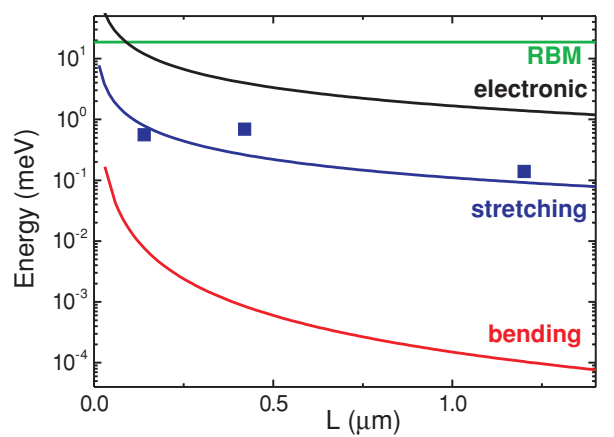

Figure 12. Energy scales of different vibrations and electronic excitations plotted on a log scale for a nanotube with $1.4 \mathrm{~nm}$ diameter. The radial breathing mode (green) does not depend on the length $L$. The bending mode vibrations (red) have a $L^{-2}$ dependence. The mean electronic level spacing (black) and the stretching mode (blue) vibrations depend inversely on the length. The symbols are excitation energy values obtained for suspended NTs with different lengths.

$1.3 \mathrm{~g} \mathrm{~cm}^{-3}, Y=1 \mathrm{TPa}$, the vibrational energy corresponding to the fundamental mode is $\sim 110 \mu \mathrm{eV} / L(\mu \mathrm{m}) .{ }^{5}$ As figure 12 shows, the data are in good agreement with these predicted values.

The coupling of electronic levels with vibrational modes (quantum harmonic oscillators) can be described in terms of the Franck-Condon model [55]. According to the FranckCondon principle, an electron in an electronic transition moves so fast that the nuclear positions are virtually the same immediately before and after the transition. As a consequence, the transition rate is proportional to the Franck-Condon factors defined as the square of the overlap integral between the vibrational wavefunctions of the two states involved. An important parameter is the electron-phonon coupling factor, $g=\frac{1}{2}\left(\frac{x}{x_{0}}\right)^{2}$. This is the ratio of the classical displacement length, $x$, to the quantum-mechanical oscillator length, $x_{0}=$ $\sqrt{\hbar / m \omega_{0}}$.

For low damping, the vibrational levels remain sharp and the Franck-Condon model predicts steps in the currentvoltage characteristics, that are equally spaced in energy (bias voltage). In the presence of strong relaxation, the normalized step heights are given by [45] $P_{n}=e^{-g} g^{n} / n$ !. In the strong coupling $(g \gg 1)$ limit, the height of the first steps is exponentially suppressed (phonon blockade) [45-47]. Multiple steps only arise if $g$ is of the order of 1 or larger and the observation of a spectrum of equally spaced excitation lines therefore indicates that the e-ph coupling in our suspended nanotubes must be rather strong.

In figure $11(b)$, the red curve represents the step heights $\left(P_{n}\right)$ given by the Franck-Condon model with strong relaxation discussed above. Considering the simplicity of the model, reasonable agreement is obtained. The comparison yields an estimate of $g$ of 0.95 . Similar values of $g$ are found for different samples with different lengths [44] indicating that it is approximately length independent. The mechanism behind this surprising high e-ph coupling is not understood [44].

5 The twisting mode vibration of the nanotube has a comparable energy with the stretching mode. However, the twisting mode does not couple [51]. 


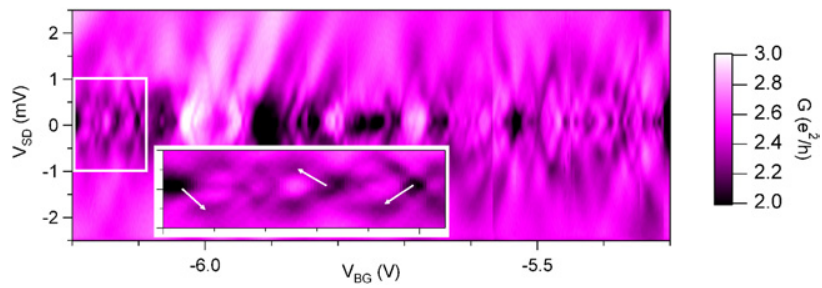

Figure 13. Differential conductance measured using a lock-in amplifier as a function of the back-gate voltage with the top-gate values such that they induce almost no barrier. Fabry-Perot-type interferences are observed over a wide gate range. The inset shows a zoom-in of the region in the white rectangle. Destructive interferences at $\pm 0.6 \mathrm{meV}$ are clearly identified (see arrows). The high conductance (up to $G=3.1 e^{2} / h$ ) shows that the Pd contacts have a transmission close to $T=1$.

\section{Excited states in carbon nanotube double quantum dots}

Double quantum dots (DQDs) are interesting systems [56] and have gained renewed interest for the possibility of realizing solid-state quantum bits [48]. Here we present lowtemperature measurements in a fully tunable CNT DQD. A new fabrication technique has been used for the top gates (TGs) in order to avoid covering the whole nanotube with an oxide layer. The TGs allow us to form single dots and control the coupling between them [49]. We observe four-fold shell filling and excited states in the DQD, a necessary step for determining the spin and orbital relaxation times.

In order to realize CNT DQDs (figure 3), it is desirable to create tuneable tunnel barriers at arbitrary locations in a CNT, and some elementary devices have already been demonstrated [57-60]. In order to make use of a CNT DQD in quantum information processing access to (spin) excited states is crucial. Narrow top gates are evaporated such that only a small portion of the CNT is covered with oxide. We show electronic transport through the ground and excited energy states of CNT DQDs.

We have fabricated several devices which we can operate in both the p-doped and n-doped regions. Only one sample will be considered here. At low temperatures $(300 \mathrm{mK})$, we observe the highest conductance when applying negative voltages to the top gates and operate the device in this holetransport regime. Figure 13 shows a differential conductance plot as a function of source-drain and back-gate voltages. The highly doped silicon substrate is used as the back gate and changes the electrochemical potential of the nanotube uniformly. Note that the average conductance is between two and three times $e^{2} / h$ (the measured maximum is $3.14 e^{2} / h$ ). The pattern in figure 13 is due to interference of the charge carriers in the nanotube (Fabry-Perot interference), which has been studied in nanotubes [6]. The pattern here is less regular. The bias voltage at the crossing point, $V_{C}$, between adjacent left- and right-sloping dark lines (see white arrows) depends on the length of the nanotube $L$ as $h v_{\mathrm{F}} / 2 e=L V_{\mathrm{C}}$. The first intersection occurs around $V_{\mathrm{C}} \sim 0.5-0.6 \mathrm{meV}$, corresponding to a length of about $3 \mu \mathrm{m}$. This suggests that the electron scattering occurs primarily at the nanotube-Pd interfaces, which are about $2 \mu \mathrm{m}$ apart, since the extracted length is much larger than the top-gate spacing $(\sim 500 \mathrm{~nm})$.
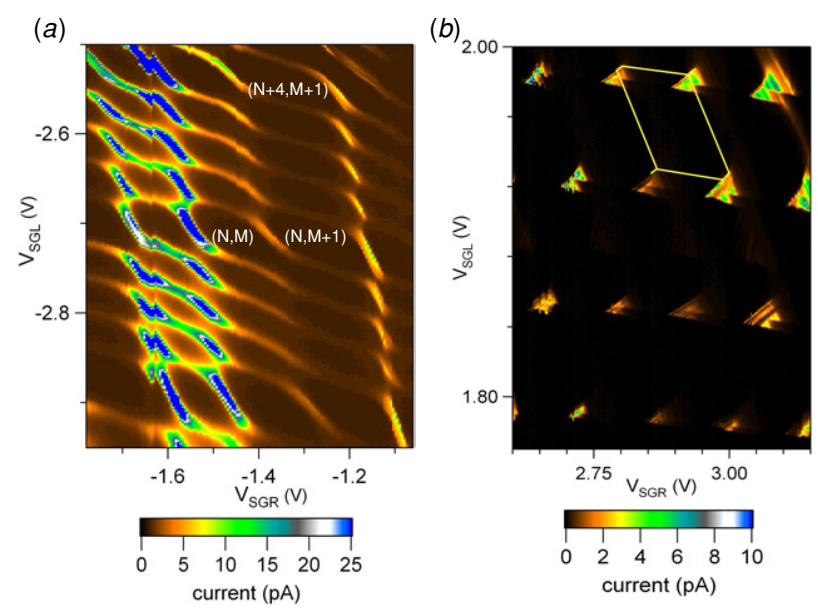

Figure 14. (a) Characteristic honeycomb pattern for the CNT DQD system in the strongly coupled regime when measured as a function of the two side gates for $1 \mathrm{mV}$ bias voltage. The four-fold shell filling of the left dot is clearly visible, from which we can identify the filled shells when the charge number is $N, N+4$, etc.

(b) Honeycomb structure of the current for the DQD in the weakly coupled regime. Triple points with excited states are visible (bias $5 \mathrm{mV}$ ).

By applying positive voltages to the top gates, we form barriers in the p-type region and can form single dots on both the left and right CNT segments individually (see figure 3). The energy level spacing, $\Delta$, we extract corresponds to the length of the CNT between top gates [49].

Figure 14(a) shows the characteristic 'honeycomb' structure of the current through a DQD [56] in the strongly coupled regime. Here, the two dots are not completely separated but interact via tunnel coupling, thus forming the analogue of a molecule with covalent bonding. The cotunnelling lines of the hexagonal pattern are visible and exhibit four-fold shell filling for the left dot: a large hexagon is followed by three small ones in the vertical direction of the left side gate. This pattern repeats for every electron number in the right dot. In figure 14(b), we show the double dot in the weakly coupled regime, i.e. the inter-dot tunnel resistance is high and the capacitive coupling between the dots dominates the transport behaviour. The triple points of the expected hexagon pattern are clearly visible and, due to the large bias, develop into overlapping triangles [56]. Excited states are observed at every triple point.

A high-resolution measurement of a pair of triple points (electron and hole cycles) [56] is shown in figure 15 for $V_{\mathrm{SD}}=4 \mathrm{mV}$. At the baseline of the triangle, the ground states of the two dots are aligned and shifted together from the Fermi level of the drain (point a in figure 15) to the Fermi level of the source (point b). At the centre of the baseline, they lie exactly in the middle between source and drain. On a line from this point to the tip of the triangle (point c), the states of the right dot are shifted downwards to the Fermi level of the source, while the states of the left dot shift upwards to the drain (a positive bias is applied at the source contact, while the drain contact is put to ground). Along this line, we see sharp excitations at $0.33,1.24,1.55$ and $1.8 \mathrm{meV}$ (see inset in figure 15). These lines belong to different excited states of the 


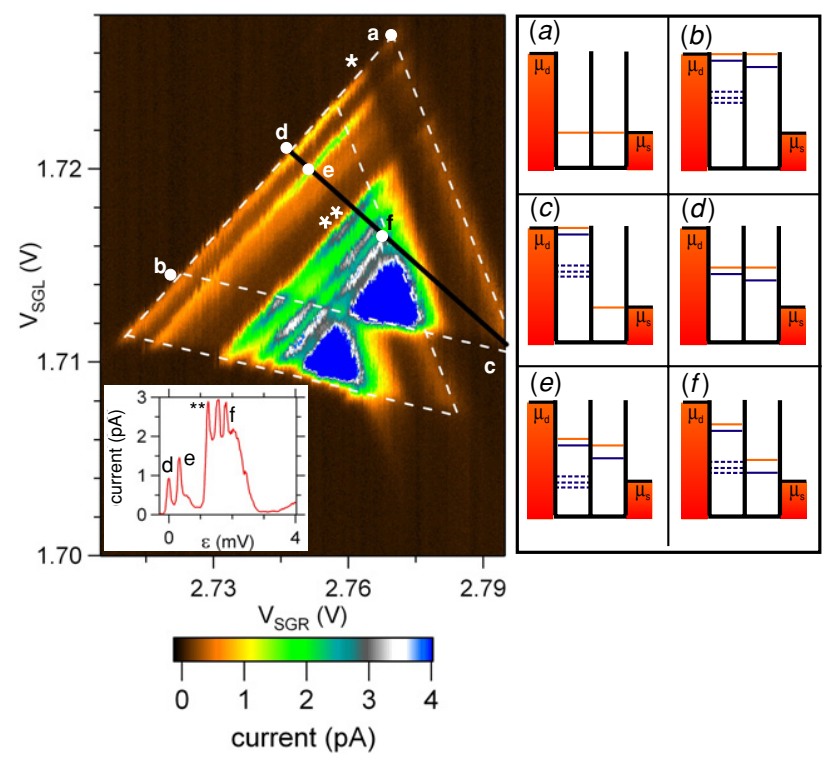

Figure 15. Current versus side-gate voltages for a typical example of a triple point pair at high bias $\left(V_{\mathrm{SD}}=4 \mathrm{mV}\right)$ in the p-doped region (hole transport). The ground-state tunnelling is weaker than tunnelling through excited states. Lines parallel to the base (point a to point $b$ ) of a triangle belong to tunnelling through excited states of the left dot, while the right dot excited states show up parallel to the upper-right side of the triangle (the first one enters the bias window at point $(*))$. The inset shows a line cut from the centre of the base of the upper triangle to the triangle tip (black line from point $\mathrm{d}$ to point $\mathrm{c}$ ), i.e., current as a function of the detuning between levels. The level schemes of the double dot corresponding to the points a-f depicted in the triangle are shown on the right-hand side of the figure. Light (orange) lines represent the ground states and dark (blue) lines the hole-excited states (the measurement is done in the p-doped region). The dashed lines belong to the next excited states corresponding to $(* *)$.

left dot which are probed by states of the right dot. An area of non-resonant current spreads between 2 and $2.8 \mathrm{meV}$.

In the following, we give a possible scenario for the resonant transport. Afterwards, we discuss the non-resonant current. On the right-hand side of the triangles of both triple points, there appears to be a region of strongly suppressed current. This feature could be explained by bad coupling of the ground state of the right dot to the source. As the levels of the dots move upwards with lowering side-gate voltages, at point $(*)$ the first hole-excited state of the right dot enters the bias window at $\sim 650 \mu \mathrm{eV}$. The coupling of this level to the source contact is stronger, thus enhancing the current.

The lines parallel to the baseline of the triangle belong to resonant transport through hole-excited states of the left dot. Only the first of these excited states at $\sim 330 \mu \mathrm{eV}$ is probed by the ground state of the right dot (point e). At the other lines, the excited state of the right dot at $\sim 650 \mu \mathrm{eV}$ is aligned with the excited states of the left dot (see point $f$ as an example). This could also explain the larger current through these lines, as the excited states are probably better coupled to the contacts. The energy splitting of the second excited state $(* *)$ of the left dot to its ground state is $1.9 \mathrm{meV}$. This fits well with the value for the level splitting we obtained from the single dot measurement. The next two lines are separated by 310 and $\sim 560 \mu \mathrm{eV}$ with respect to the level $(* *)$ at $\Delta$.
Like the first one at $\sim 330 \mu \mathrm{eV}$ (point e), they are comparable in size with the low energy splitting found in the single dot measurement of the left QD.

Non-resonant transport can occur if an electron loses energy due to spontaneous emission of an acoustic phonon [61]. However, we do not observe the expected decay of the current for one-dimensional acoustic phonons with detuning of the DQD states. The non-resonant current between $2 \mathrm{meV}$ and $2.8 \mathrm{meV}$ seems to have its origin rather in level broadening of excited states at higher energy.

Electron-phonon coupling in a molecule such as a CNT can show up as sharp resonance lines. These would be equidistant with an energy difference that depends on the diameter and length of the tube [44]. For a length of $2 \mu \mathrm{m}$, an energy difference of $E_{\text {phonon }} \sim 55 \mu \mathrm{eV}$ is expected for the stretching mode [44]. If the size of the single QDs of $\sim 500 \mathrm{~nm}$ determined the energy of the phonons, one would expect $E_{\text {phonon }} \sim 440 \mu \mathrm{eV}$. None of these energy scales show up in the lines inside the triangle. Thus, we conclude that the lines inside the triangle are due to resonant transport through electronic excitations.

\section{Acknowledgments}

We thank P Beliczynski, Ya M Blanter, C Dekker, $\mathrm{S}$ De Franceschi, J Kong and C Meyer for their help. Financial support is obtained from the Dutch organization for Fundamental Research on Matter (FOM), which is financially supported by the 'Nederlandse Organisatie voor Wetenschappelijk Onderzoek' (NWO).

\section{References}

[1] Iijima S 1991 Nature 35456

[2] For reviews, see Dekker C 1999 Phys. Today 5222 McEuen P L 2000 Phys. World 1331

[3] Tans S J, Devoret M H, Dai H J, Thess A, Smalley R E, Geerligs L J and Dekker C 1997 Nature 386474

[4] Bockrath M, Cobden D H, McEuen P L, Chopra N G, Zettl A, Thess A and Smalley R E 1997 Science 2751922

[5] Bockrath M, Cobden D H, Lu J, Rinzler A G, Smalley R E, Balents L and McEuen P L 1999 Nature 397598

[6] Liang W, Bockrath M, Bozovic D, Hafner J H, Tinkham M and Park H 2001 Nature 411665

[7] Nygard J, Cobden D H and Lindelof P E 2000 Nature 408342

[8] Jarillo-Herrero P, Kong J, van der Zant H S J, Dekker C, Kouwenhoven L P and De Franceschi S 2005 Nature 434484

[9] LeRoy B J, Lemay S G, Kong J and Dekker C 2004 Nature 432371

[10] Sazonova V, Yaish Y, Ustunel H, Roundy D, Arias T A and McEuen P L 2004 Nature 431284

[11] Morpurgo A F, Kong J, Marcus C M and Dai H 1999 Science 286263

[12] Kasumov A Y, Deblock R, Kociak M, Reulet B, Bouchiat H, Khodos I I, Gorbatov Y B, Volkov V T, Journet C and Burghard M 1999 Science 2841508

[13] Jarillo-Herrero P, van Dam J A and Kouwenhoven L P 2006 Nature 439953

[14] Javey A, Guo J, Wang Q, Lundstrom M and Dai H 2003 Nature 424654

[15] Bronikowski M J, Willis P A, Colbert D T, Smith K A and Smalley R E 2001 J. Vac. Sci. Technol. A 191800

[16] Fuhrer M S et al 2000 Science 288494

[17] Nygård J and Cobden D H 2001 Appl. Phys. Lett. 794216 
[18] Bachtold A, Hadley P, Nakanishi T and Dekker C 2001 Science 2941317

[19] Park J and McEuen P L 2001 Appl. Phys. Lett. 791363

[20] Kouwenhoven L P, Austing D G and Tarucha S 2001 Rep. Prog. Phys. 64701

[21] Dresselhaus M S, Dresselhaus G and Eklund P C 1996 Science of Fullerenes and Carbon Nanotubes (San Diego, CA: Academic)

[22] Postma H W C, Sellmeijer A and Dekker C 2000 Adv. Mater. 121299

[23] Jarillo-Herrero P, Sapmaz S, Dekker C, Kouwenhoven L P and van der Zant H S J 2004 Nature $\mathbf{4 2 9} 389$

[24] Cobden D H and Nygård J 2002 Phys. Rev. Lett. 89046803

[25] Heinze S, Tersoff J, Martel R, Derycke V, Appenzeller J and Avouris P 2002 Phys. Rev. Lett. 89106801

[26] Grabert H and Devoret M H 1992 Single Charge Tunneling (New York: Plenum)

[27] Park H, Park J, Lim A K L, Anderson E H, Alivisatos A P and McEuen P L 2000 Nature 40757

[28] Wigner E 1934 Phys. Rev. 461002

[29] Hausler W and Kramer B 1993 Phys. Rev. B 4716353

[30] Liang W, Bockrath M and Park H 2002 Phys. Rev. Lett. 88126801

[31] Babic B and Schönenberger C 2004 Phys. Rev. B 70195408

[32] Sapmaz S, Jarillo-Herrero P, Kong J, Dekker C, Kouwenhoven L P and van der Zant H S J 2005 Phys. Rev. B 71153402

[33] Moriyama S, Fuse T, Suzuki M, Aoyagi Y and Ishibashi K 2005 Phys. Rev. Lett. 94186806

[34] Makarovski A, Zhukov A, An L, Liu J and Finkelstein G 2005 Persistent orbital degeneracy in carbon nanotubes Preprint cond-mat/0508401

[35] Oreg Y, Byczuk K and Halperin B I 2000 Phys. Rev. Lett. 85365

[36] Lemay S G, Janssen J W, van den Hout M, Mooij M, Bronikowski M J, Willis P A, Smalley R E, Kouwenhoven L P and Dekker C 2001 Nature 412617

[37] Ajiki H and Ando T 1993 J. Phys. Soc. Japan 622470

[38] Minot E D, Yaish Y, Sazonova V and McEuen P L 2004 Nature $\mathbf{4 2 8} 536$

[39] Tarucha S, Austing D G, Honda T, van der Hage R J and Kouwenhoven L P 1996 Phys. Rev. Lett. 773613

[40] Jarillo-Herrero P, Kong J, van der Zant H S J, Dekker C, Kouwenhoven L P and De Franceschi S 2005 Phys. Rev. Lett. 94156802
[41] Sasaki S, De Franceschi S, Elzerman J M, van der Wiel W G, Eto M, Tarucha S and Kouwenhoven L P 2000 Nature 405764

[42] De Franceschi S, Sasaki S, Elzerman J M, van der Wiel W G, Tarucha S and Kouwenhoven L P 2001 Phys. Rev. Lett. 86878

[43] Kogan A, Amasha S, Goldhaber-Gordon D, Granger G, Kastner M A and Shtrikman H 2004 Phys. Rev. Lett. 93166602

[44] Sapmaz S, Jarillo-Herrero P, Blanter Y M, Dekker C and van der Zant H S J 2006 Phys. Rev. Lett. 96026801

Sapmaz S, Jarillo-Herrero P, Blanter Y M and van der Zant H S J 2005 New J. Phys. 7243

[45] Braig S and Flensberg K 2003 Phys. Rev. B 68205324

[46] Mitra A, Aleiner I and Millis A J 2004 Phys. Rev. B 69245302

[47] Koch J and von Oppen F 2005 Phys. Rev. Lett. 94206804

[48] Loss D and DiVincenzo D P 1998 Phys. Rev. A 57120

[49] Sapmaz S, Meyer C, Beliczynski P, Jarillo-Herrero P and Kouwenhoven L P 2006 Nano Lett. 61350

[50] Dresselhaus M S and Eklund P C 2000 Adv. Phys. 49705

[51] Suzuura H and Ando T 2002 Phys. Rev. B 65235412

[52] De Martino A and Egger R 2003 Phys. Rev. B 67235418

[53] Sapmaz S, Blanter Y M, Gurevich L and van der Zant H S J 2003 Phys. Rev. B 67235414

[54] Landau L D and Lifshitz E M 1986 Theory of Elasticity (Oxford: Pergamon)

[55] Schatz G C and Ratner M A 1993 Quantum Mechanics in Chemistry (Englewood Cliffs, NJ: Prentice-Hall)

[56] van der Wiel W G, De Franceschi S, Elzerman J M, Fujisawa T, Tarucha S and Kouwenhoven L P 2003 Rev. Mod. Phys. 751

[57] Mason N, Biercuk M J and Marcus C M 2004 Science 303655

[58] Biercuk M J, Garaj S, Mason N, Chow J M and Marcus C M 2005 Nano Lett. 51267

[59] Gräber M R, Coish W A, Hoffmann C, Weiss M, Furer J, Oberholzer S, Loss D and Schönenberger C 2006 Phys. Rev. B 74075427

[60] Jørgensen H I, Grove-Rasmussen K, Hauptmann J R and Lindelof P E 2006 A single wall carbon nanotube double quantum dot Preprint cond-mat/0606374

[61] Fujisawa T, Oosterkamp T H, van der Wiel W G, Broer B W, Aguado R, Tarucha S and Kouwenhoven L P 1998 Science 282932 